\title{
Avaliação do ensemble de parametrizações físicas do modelo MM5 no evento de precipitação intensa ocorrido entre os dias 05 e 06 de abril de 2010 no município do Rio de Janeiro
}

Evaluation of the ensemble of physical parameterizations of the MM5 model in the event of intense rainfall occurred between the $5^{\text {th }}$ and the $6^{\text {th }}$ of april of 2010 in the Rio de Janeiro city

\author{
Clarice B M Lira ${ }^{* 1}$ e Marcio Cataldi ${ }^{* * 2}$ \\ 1,2 Departamento de Engenharia Agrícola e do Meio Ambiente, Universidade Federal Fluminense, Niterói - RJ, \\ Brasil \\ *clarice.lira1@gmail.com; **marcio.cataldi@gmail.com
}

\begin{abstract}
Resumo
O Município do Rio de Janeiro é atingido frequentemente por sistemas meteorológicos que causam chuvas intensas, causando transtornos econômicos e sociais de magnitude significativa. Sendo assim, o presente trabalho realiza uma análise do evento meteorológico ocorrido nos dias 05 e 06 de abril de 2010 na cidade do Rio de Janeiro, utilizando modelagem numérica via modelo MM5 (PSU/NCAR MesoscaleModel). Entre as $20 \mathrm{~h}$ do dia 05 e às $8 \mathrm{~h}$ do dia 06 choveu em média $178 \mathrm{~mm}$ neste município, praticamente o dobro do esperado para todo o mês de abril. No estudo, oito grupos de parametrizações foram utilizados, com diferentes configurações de microfísica de nuvens, cúmulos e camada limite atmosférica. Foram realizadas duas rodadas, inicializadas em horários diferentes, sendo os resultados das simulações comparados com os dados de precipitação observada, obtidos através da rede pluviométrica do Sistema Alerta-Rio. Dessa forma, foi possível analisar a eficácia do modelo para prognosticar eventos meteorológicos extremos, já que algumas configurações do modelo conseguiram capturar a intensidade elou a posição dos núcleos mais intensos de precipitação apresentando, em alguns casos, uma defasagem temporal de 3 horas. Desta forma, entende-se que este sistema prognóstico se mostra como uma ferramenta útil para prever e auxiliar na minimização das consequências dos eventos de chuvas intensas no município do Rio de Janeiro.
\end{abstract}

Palavras-chave: MM5. Ensemble de parametrizações físicas. Eventos severos.

\begin{abstract}
The city of Rio de Janeiro is often affected by meteorological systems that cause intense rainfalls. These events bring about economic and social disruptions of significant magnitude. This paper analyses the meteorological event occurred between the 05th and 06th of April 2010 in the city of Rio de Janeiro, using numerical modelling by MM5 model (PSU/NCAR Mesoscale Model). From 20h of the 5th until 8h of the 6th there was on average $178 \mathrm{~mm}$ of rainfall in the this city, almost twice the expected for April, bringing about floods, landslides, and many deaths. In this study, eight parameterization groups were used, with different configurations of cloud microphysics, cumulus and atmospheric boundary layer. Two simulation rounds were performed, starting at different times and the simulation results were compared with the observed precipitation data, obtained from pluviometric stations from the "Alerta-Rio" system. Thus, it was possible to analyse the effectiveness of the MM5 model for prognosticate extreme rainfall events as some of the model configurations have captured the position and/or intensity of the cores with intense rainfall with a three-hour time gap in some cases. Therefore, this prognostic model had proven that it can be a useful tool to forecast and reduce the consequences of extreme rainfall events in Rio de Janeiro city.
\end{abstract}

Keywords: MM5. Ensemble of physical parameterizations. Extreme events. 


\section{Introdução}

O Município do Rio de Janeiro é atingido frequentemente por sistemas meteorológicos que causam chuvas intensas. Um desses sistemas ocorreu durante os dias 05 e 06 de abril de 2010, afetando parte do Estado do Rio de Janeiro, ocasionando inundações e deslizamentos de terra principalmente nos municípios do Rio de Janeiro e de Niterói, onde mais de 200 pessoas morreram. O evento esteve associado com a passagem de um sistema frontal e acarretou um dos maiores totais de precipitação acumulada em $24 \mathrm{~h}$ e uma das maiores enchentes na cidade do Rio de Janeiro nos últimos 100 anos (REBELLO et al, 2012). Segundo a Geo-Rio, a média pluviométrica histórica para este mês é de 90 $\mathrm{mm}$ na cidade. No entanto, no acumulado entre os dia 05 e 06 de abril choveu, em média nas estações da Geo-Rio, cerca de 178 mm. Em 14 horas, choveu o dobro da média histórica de abril no Rio de Janeiro.

Para intensificar as enchentes, houve uma combinação do índice pluviométrico com o elevado nível de maré observado, dificultando o escoamento de água nas localidades mais litorâneas (COELHO et al., 2011).

Uma das formas de diminuir os prejuízos econômicos e as perdas de vidas geradas pelos desastres naturais é através do prognóstico dos fenômenos atmosféricos que ocorrem em diversas escalas meteorológicas, através de modelos numéricos de previsão do tempo, por exemplo. Esses modelos podem ser de previsão global ou modelos de mesoescala e de área limitada, sendo este último uma ferramenta indispensável aos centros de previsão e para gerar alertas. Esses modelos regionais podem fazer o "downscaling" de previsões obtidas por modelos globais e assim, reproduzir com maior detalhamento espacial os campos atmosféricos na região de interesse (PADILHA, 2011).

O modelo de previsão utilizado e avaliado nesse estudo é o MM5 (Fifth-Generation Penn State/NCAR Mesoscale Model), desenvolvido no final da década de 70 pela Pensylvania State University e pelo National Center for Atmospheric Research (NCAR) como um modelo de mesoescala comunitário. É um modelo de área limitada, dinâmica nãohidrostática, com código numérico organizado em módulos e que utiliza um sistema de coordenadas sigma que segue a topografia do terreno. Ele possui a capacidade para múltiplos aninhamentos de grade com interação bidirecional, assimilação de dados em quatro dimensões e várias opções de parametrizações físicas (GRELL et al, 1994).

Como todo modelo de área limitada, são necessárias condições iniciais e de contorno. Além disso, ele utiliza a grade Arakawa-B onde as variáveis vetoriais (componentes $\mathrm{u}$ e $\mathrm{v}$ do vento e força de Coriolis) são resolvidas nos pontos de grade, enquanto as variáveis escalares são resolvidas no interior da célula da grade. No MM5 todas as quatro fronteiras possuem campos de vento horizontais, temperatura e umidade especificados (DUDHIA et al, 2002).

No presente estudo o modelo regional MM5 foi utilizado para simular em evento de precipitação intensa utilizando oito conjuntos de parametrizações físicas, através de duas rodadas inicializadas em horários diferentes, sendo os resultados comparados com os dados de precipitação observada, provenientes da rede de estações pluviométricas do Sistema Alerta-Rio, da Fundação Geo-Rio, visando assim, avaliar a eficácia do modelo na previsão desse evento de precipitação intensa dos dias 05 e 06 de abril de 2010.

\section{Aplicações do modelo MM5}

O modelo MM5 é utilizado globalmente para modelagem em diversas situações. Dodla (2010) utilizou esse modelo numérico acoplado com nudging para simular um evento de precipitação intensa ocorrido na costa oeste da Índia no dia 26 de julho de 2005, onde foram observados totais de precipitação da ordem de 900-1000 mm na região norte da cidade de Mumbai. Os melhores resultados de precipitação produziram uma precipitação em $24 \mathrm{~h}$ de $550 \mathrm{~mm}$ nessa mesma região. Queen (2008) utilizou o modelo para simular a deposição úmida de espécies químicas, 
utilizando diferentes opções de microfísica e resoluções de grades no período de agosto a dezembro de 2002 no Estado de Carolina do Norte (EUA). A maior parte dos resultados de precipitação mensal média foram subestimados e os esquemas de microfísica que apresentaram melhores resultados foram Reisner 2 e Dudhia. Já Zhang (2011), para estudar a poluição do ar na China, utilizou o MM5 para simular condições meteorológicas durante seis meses entre 2007 e 2008, utilizando três domínios acoplados, abrangendo a região leste da Ásia, a região leste da China e a província de Shandong, com resolução horizontal de 36, 12 e $4 \mathrm{~km}$, respectivamente. Foi observado que as previsões meteorológicas são mais coincidentes com as observações em regiões urbanas. Os resultados da modelagem em regiões costeiras ou montanhosas foram piores devido à complexidade da topografia, influencia do efeito estufa vindo das cidades, brisas marinhas/terrestres e grandes altitudes. Amengual (2008) fez um estudo hidrometeorológico utilizando os resultados de precipitação gerados pelo MM5 durante quatro eventos de precipitação intensa ocorridos na bacia hidrográfica de Albufera, localizada em Majorca, Espanha. Esses resultados foram utilizados para simular vazões através de um modelo hidrológico (HEC-HMS) e comparados com os resultados de vazões gerados com a precipitação observada. No estudo foi utilizado um ensemble de parametrizações físicas, a fim de tentar reproduzir todos os processos responsáveis pela geração de eventos de precipitação intensa. Os autores concluíram que as simulações conseguiram reproduzir os eventos de precipitação intensa na região, permitindo a utilização dos resultados para simular vazões nessa bacia. Bray (2011) estudou a utilização do MM5 para gerar estimativas de precipitação para estudos hidrológicos em locais onde existe pouca informação de precipitação observada. O estudo examinou a incerteza dos resultados de vazão gerados pela precipitação simulada no MM5, variando o tamanho do domínio e o espaçamento entre as grades aninhadas. Os resultados mostraram que esses parâmetros tiveram um impacto significativo nos resultados do modelo chuvavazão. Oyama (2006) realizou simulações climáticas utilizando o modelo regional MM5 em baixa resolução horizontal $(250 \mathrm{~km})$, a fim de verificar se o modelo conseguiria representar realisticamente o clima da região tropical da América do Sul. Em relação aos valores médios anuais, o MM5 conseguiu representar o padrão geral de precipitação da região tropical da América do Sul. No entanto, regionalmente, houveram erros sistemáticos, a saber: o MM5 superestimou (subestimou) a precipitação no nordeste, noroeste e oeste (sudeste) da América do Sul. Jerez (2013) mostrou a sensibilidade das mudanças na simulação do clima regional de acordo com a escolha das parametrizações físicas. A modelagem ocorreu na Península Ibérica durante o período presente (1970-1999) e futuro (2070-2099), utilizando ensemble de oito conjuntos de parametrizações. Os resultados mostraram grande variação nas condições sinóticas que impulsionam os resultados do modelo, despontando a necessidade de um melhor entendimento dos processos parametrizados nas sub-grades do modelo. Fernandez (2007) também analisou a sensibilidade de diferentes conjuntos de parametrizações físicas em simulações utilizando o MM5, durante vários períodos de cinco anos na região da Península Ibérica, comparando as simulações com a precipitação observada e dados de temperatura da superfície. Os resultados mostraram que os ciclos anuais de precipitação e temperatura da superfície foram bem reproduzidos, porém nenhum conjunto de parametrizações foi considerado melhor ao simular tanto a precipitação quanto temperatura de superfície, em todas as estações e sub-regiões.

\section{Evento de Precipitação Intensa}

O evento ocorreu em parte do Estado do Rio de Janeiro, afetando a região metropolitana e serrana, porém, esse estudo foca no município do Rio de Janeiro.

O relevo acidentado e diversificado da cidade contribui para a grande variabilidade espacial da precipitação total média anual, com um índice pluviométrico entre 1000 a $1500 \mathrm{~mm}$ anuais (MACHADO, 2009). De acordo com o estudo de MACHADO (2009), a partir do conjunto de 10 anos de dados (1997-2006) coletados em 30 estações pluviométricas da Fundação Geo-Rio, os máximos de precipitação ao longo do ano e 
sazonalmente localizam-se sobre os três maciços existentes na cidade: maciço de Gericino, maciço da Pedra Branca e maciço da Tijuca. Além disso, o padrão de distribuição espacial de precipitação, com máximo de precipitação a barlavento e mínimos a sotavento das serras, é explicado pelos sistemas meteorológicos em deslocamento preferencialmente de sudeste para nordeste. Tal processo é amplificado pelo levantamento de ar úmido trazido pela brisa marítima que predomina no município. O trimestre mais chuvoso é de novembro a janeiro enquanto que o menos chuvoso é de junho a agosto.

Para o estudo do evento de precipitação intensa ocorrido, foram utilizados dados observados de precipitação, provenientes da rede de 30 estações pluviométricas do Sistema Alerta-Rio, da Geo-Rio, durante o período de estudo, entre 05/04/2010 e 06/04/2010 (Tabela 1).

Tabela 1 - Estações pluviométricas e localização

\begin{tabular}{|c|c|c|c|}
\hline Número & Estação pluviométrica & Latitude & Longitude \\
\hline 1 & SANTA TERESA & -22.9317 & -43.1964 \\
\hline 2 & COPACABANA & -22.9864 & -43.1894 \\
\hline 3 & ILHA DO GOVERNADOR & -22.8181 & -43.2103 \\
\hline 4 & JARDIM BOTÂNICO & -22.9728 & -43.2239 \\
\hline 5 & ROCINHA & -22.9858 & -43.245 \\
\hline 6 & SAÚDE & -22.8981 & -43.1944 \\
\hline 7 & TIJUCA & -22.9319 & -43.2217 \\
\hline 8 & URCA & -22.9558 & -43.1667 \\
\hline 9 & VIDIGAL & -22.9925 & -43.2331 \\
\hline 10 & ANCHIETA & -22.8269 & -43.4033 \\
\hline 11 & AV.BRASIL/MENDANHA & -22.8569 & -43.5411 \\
\hline 12 & BANGU & -22.8803 & -43.4658 \\
\hline 13 & CAMPO GRANDE & -22.9036 & -43.5619 \\
\hline 14 & JACAREPAGUÁ/CIDADE DE DEUS & -22.9456 & -43.3628 \\
\hline 15 & GRAJAÚ & -22.9222 & -43.2675 \\
\hline 16 & GRANDE MÉIER & -22.8906 & -43.2781 \\
\hline 17 & GROTA FUNDA & -23.0144 & -43.5194 \\
\hline 18 & GUARATIBA & -23.0503 & -43.5947 \\
\hline 19 & IRAJÁ & -22.8269 & -43.3369 \\
\hline 20 & BARRA/ITANHANGÁ & -22.9983 & -43.3022 \\
\hline 21 & LARANJEIRAS & -22.9406 & -43.1875 \\
\hline 22 & MADUREIRA & -22.8733 & -43.3389 \\
\hline 23 & PENHA & -22.8444 & -43.2753 \\
\hline 24 & PIEDADE & -22.8931 & -43.3072 \\
\hline 25 & RECREIO DOS BANDEIRANTES & -23.01 & -43.4406 \\
\hline 26 & BARRA/RIO CENTRO & -22.98 & -43.4103 \\
\hline 27 & SANTA CRUZ & -22.9094 & -43.6844 \\
\hline 28 & SÃO CRISTÓVÃO & -22.8967 & -43.2217 \\
\hline 29 & SEPETIBA & -22.9689 & -43.7117 \\
\hline 30 & JACAREPAGUÁ/TANQUE & -22.9125 & -43.3647 \\
\hline
\end{tabular}

A Figura 1 mostra a topografia da cidade do Rio de Janeiro com seus principais maciços e a distribuição espacial dos postos pluviométricos da Geo-Rio.
Para analisar o evento de precipitação foram feitos gráficos, utilizando a variável de precipitação acumulada a cada 3 , durante o período de estudo. O primeiro gráfico (Figura 2) mostra os totais de precipitação acumulada a cada 3 horas em cada estação e a média de todas as estações (linha mais grossa). É notável o período de início da chuva, em torno das $15 \mathrm{~h}$ do dia 05 de abril. A partir daí, houve um aumento expressivo da precipitação acumulada a cada $3 \mathrm{~h}$, com os períodos de pico às $21 \mathrm{~h}$ do dia 05 de abril e às 03h do dia 06 de abril de 2010, com uma precipitação média de $40 \mathrm{~mm}$. A partir de $12 \mathrm{~h}$ do dia 06 de abril, a chuva diminuiu consideravelmente, com alguns postos já não apresentando mais chuva, ficando a precipitação média em torno de $5 \mathrm{~mm}$. O posto que acumulou mais chuva em 3 h foi o posto 23 (Penha) com quase $90 \mathrm{~mm}$ de precipitação entre $00 \mathrm{~h}$ e $03 \mathrm{~h}$ do dia 06 de abril.

É importante ressaltar que a precipitação permaneceu no dia 07 de abril, com bem menos quantidade de chuva, porém no presente estudo só estão sendo analisados os dias mais críticos, sendo estes, 05 e 06 de abril.

Para avaliar o acúmulo de precipitação total durante os dias do estudo é preciso observar a Figura 3. Percebe-se que entre às $15 \mathrm{~h}$ do dia 05 de abril e $00 \mathrm{~h}$ do dia 07 de abril, foram acumulados, em média, quase $240 \mathrm{~mm}$ de chuva. A estação com maior acumulo de chuva foi Rocinha (5), com mais de $350 \mathrm{~mm}$ de chuva.

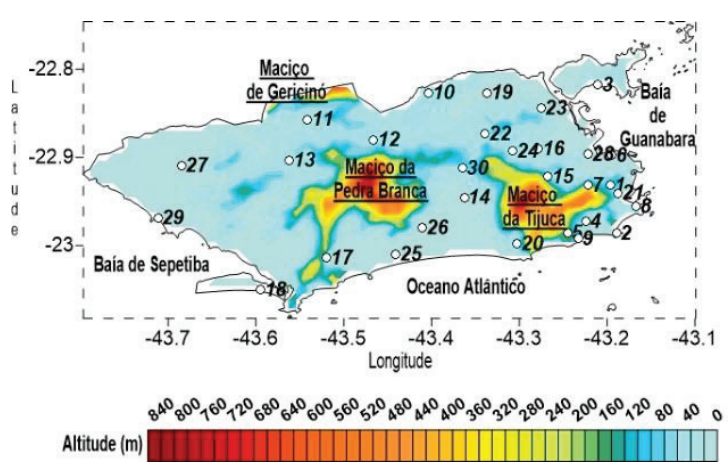

Figura 1- Topografia e distribuição espacial dos postos da Geo-Rio no município do Rio de Janeiro.

Fonte dos dados de topografia: $<h t t p: / / w w w . s r c . c o m />$ 


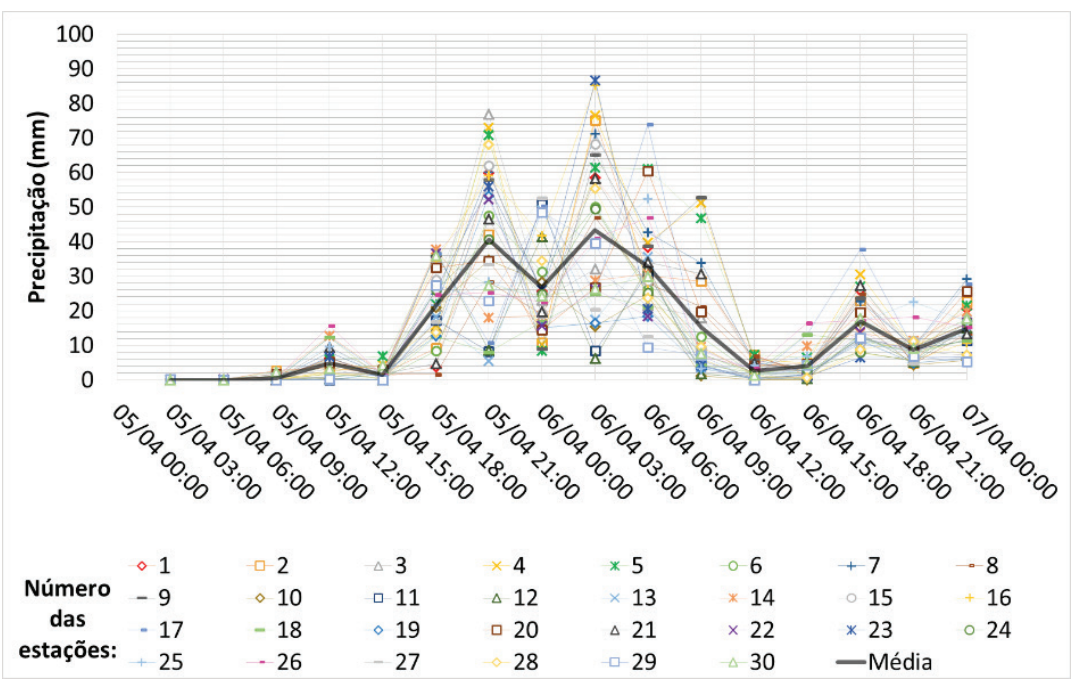

Figura 2 - Valores de precipitação acumulados a cada 3 horas entre os dias 05 e 06 de abril de 2010

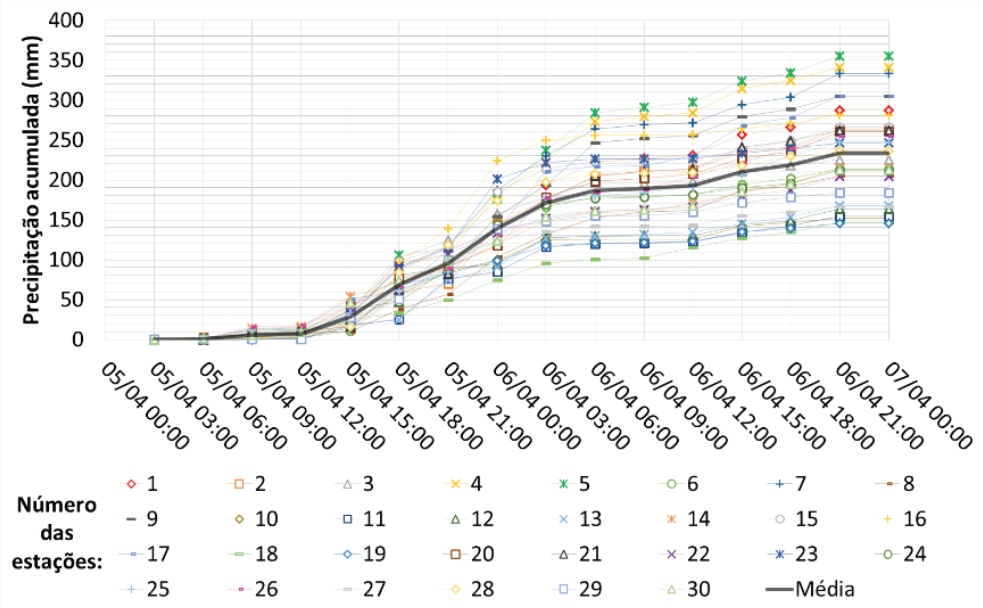

Figura 3 - Valores acumulados de precipitação entre os dias 5 e 6 de abril de 2010

\section{Análise sinótica}

De acordo com a síntese sinótica mensal do mês de abril de 2010, apresentada no sítio de internet do CPTEC, Centro de Previsão de Tempo e Estudos Climáticos, $(<$ http://wwww.cptec.inpe.br/noticias/faces/noticias.jsp ? idConsulta $=$ EidQuadros=109>) o evento começou no dia 05 de abril com a incursão de uma frente fria ao Rio de Janeiro. Ela se intensificou a partir de um cavado de altos níveis vindo de outra frente fria que estava atuando na Argentina, e que se associou rapidamente ao sistema frontal que estava no estado do Rio de Janeiro.

Esta frente fria, formada a partir do deslocamento de uma massa de ar úmido e fria (marítima), se encontrou com a massa de ar úmido e instável que já se encontrava sobre o
Sudeste, gerando um grande contraste térmico, intensificado pelo relevo da cidade do Rio de Janeiro (disposição da cadeia de montanhas na direção SW/NE). E essa combinação provocou um evento meteorológico extremo, colaborando para uma grande intensidade de precipitação. (PADILHA, 2011). As nuvens geradas foram nuvens estratiformes oriundas de convecção rasa, ocasionando uma precipitação constante e de intensidade moderada e em alguns momentos forte.

Através das imagens obtidas pelo radar do Pico do Couto (RJ) da REDEMET (Rede de Meteorologia do Comando da Aeronáutica), CaPPI $400 \mathrm{~km}$, e das imagens infravermelhas realçadas obtidas pelo satélite GOES-12, do banco de dados do CPTEC, é possível observar visualmente o que aconteceu nos momentos de 
início de chuva ( $18 Z$ do dia 05 de abril), de pico (00Z do dia 06 de abril) e de diminuição da chuva (15Z do dia 06 de abril).

Observando as imagens de radar (Figura 4), verifica-se que às $18 \mathrm{Z}$ do dia 05 a chuva começa a entrar no município do Rio de Janeiro e às $00 \mathrm{Z}$ do dia 06 nota-se a concentração da precipitação no sul do Estado. O radar indica zonas de ecos esparsos tipo estratiforme com intensidade variando de 35 a $50 \mathrm{dBZ}$ (moderado a forte). Já às $15 Z$ do dia 06 a chuva se encontra deslocada para o oceano, ao Sul do Estado, com intensidade de 15 a $30 \mathrm{dBZ}$ (Muito fraco a moderado).

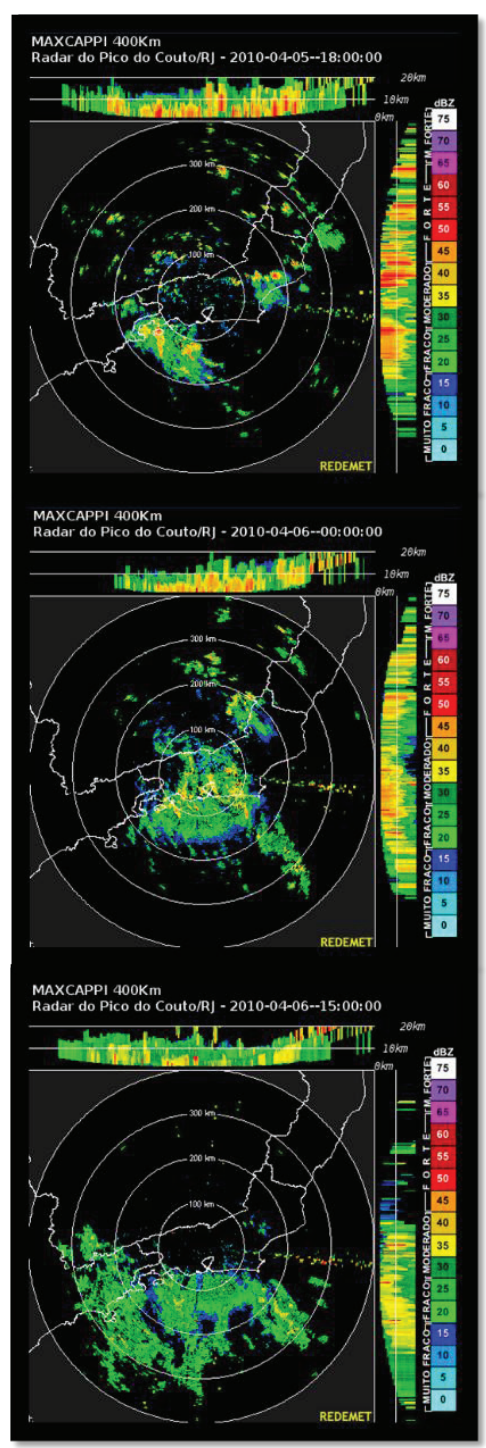

Figura 4 - Imagens do radar da REMET, de cima para baixo, às $18 Z$ do dia 05/04, 00Z do dia 06/04 e 15Z do dia 06/04.

Fonte: REDEMET.

No momento de início da chuva, $18 \mathrm{Z}$ do dia 05 de abril, na imagem de satélite (Figura 5) notam-se nuvens esparsas no Estado do Rio, associadas com a frente fria, e algumas áreas de instabilidade nos estados de Santa Catarina e Paraná, associadas com um cavado em níveis médios, que posteriormente fecha a sua circulação formando um vórtice. Já às $00 \mathrm{Z}$ do dia 06/04, a frente fria se localiza principalmente nos municípios do Rio de Janeiro e de Niterói. Porém, percebe-se que a maior parte das nuvens são rasas a médias. A partir das $15 \mathrm{Z}$ do dia 06 de abril, observa-se o afastamento da frente para o oceano Atlântico, e a aproximação do cavado vindo da Argentina.

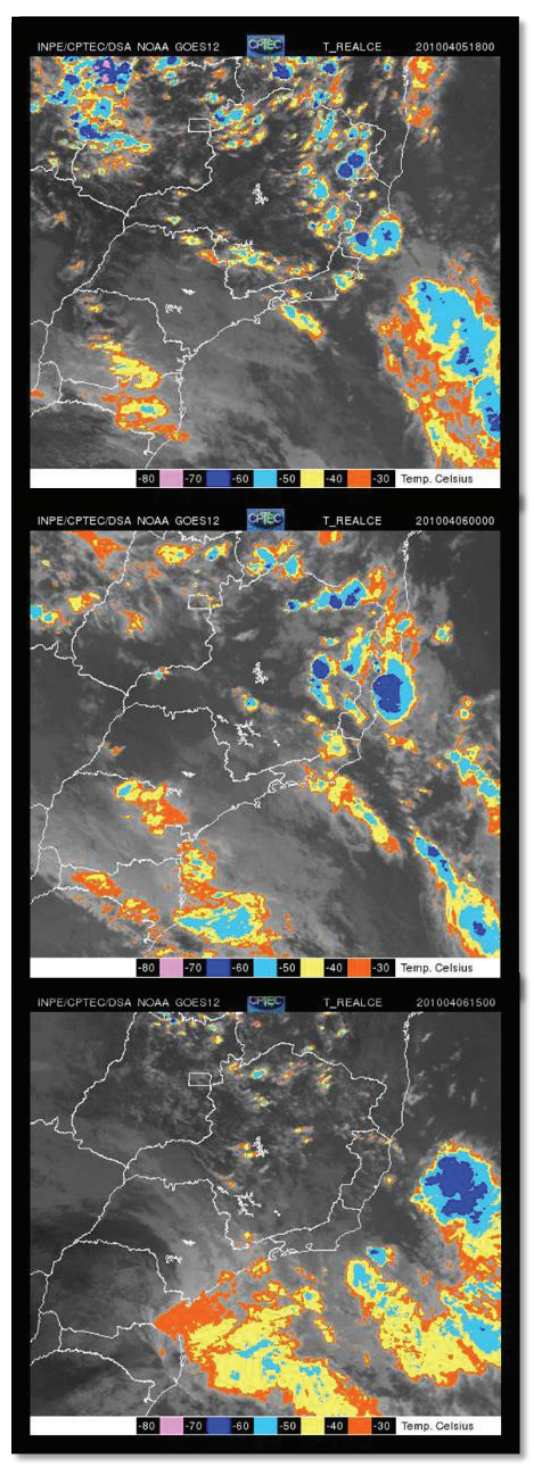

Figura 5 - Imagens do satélite GOES-12, de cima para baixo, às $18 Z$ do dia $05 / 04,00 Z$ do dia $06 / 04 \mathrm{e}$ $15 Z$ do dia 06/04.

Fonte: CPTEC/INPE

\section{Dados e Metodologia}




\subsection{Dados pluviométricos observados}

Através dos dados de precipitação já citados na seção 3, foram gerados mapas da precipitação acumulada a cada $3 \mathrm{~h}$ do município do Rio de Janeiro, utilizados na comparação com as imagens geradas pelo modelo MM5.

Além disso, foi gerado um mapa da precipitação acumulada em $24 \mathrm{~h}$ a partir de $09 \mathrm{~h}$ (local) do dia 05/04/2010 até às 09h (local) do dia 06/04/2010, sendo este o instrumento de comparação com as previsões de $24 \mathrm{~h}$ geradas pelas diferentes parametrizações do modelo MM5.

\subsection{Simulações do modelo MM5}

Nas simulações desenvolvidas utilizaram-se três domínios aninhados, sendo o primeiro com resolução de $27 \mathrm{~km}$, abrangendo a região Sudeste e parte das regiões Sul e Centro-Oeste, o segundo com resolução de $9 \mathrm{~km}$, abrangendo o Estado do Rio de Janeiro e parte de São Paulo e o terceiro com resolução de $3 \mathrm{~km}$, abrangendo a cidade de Niterói, Rio de Janeiro e parte da região serrana. A configuração "two-way" não é usada, ou seja, as informações só passam da grade maior para menor. Em todos os domínios foram utilizados dados de topografia e elevação do solo da United State Geological Survey (USGS) para iniciar o primeiro módulo do modelo (TERRAIN). Os dados de uso do solo também são originários da USGS e possuem resolução espacial de $1 \mathrm{~km}$.

Para cada simulação do modelo, oito grupos de parametrizações foram utilizados, com diferentes configurações de microfísica de nuvens, cumulus e camada limite atmosférica (Tabela 2). As escolhas de cada conjunto foram desenvolvidas ao longo do projeto Tempo Niterói desenvolvido na Universidade Federal Fluminense

\section{(<http://www.temponiteroi.uff.br/projeto.html $>$ ).}

Foram utilizados 30 níveis na vertical com aproximadamente 15 níveis verticais até $850 \mathrm{hPa}$.

Duas rodadas foram realizadas: na rodada 1 , o modelo foi inicializado com dados meteorológicos do National Centers for Environment Prediction (NCEP) do dia 04 de

Tabela 2 - Configurações dos oito grupos de parametrizações utilizados no modelo

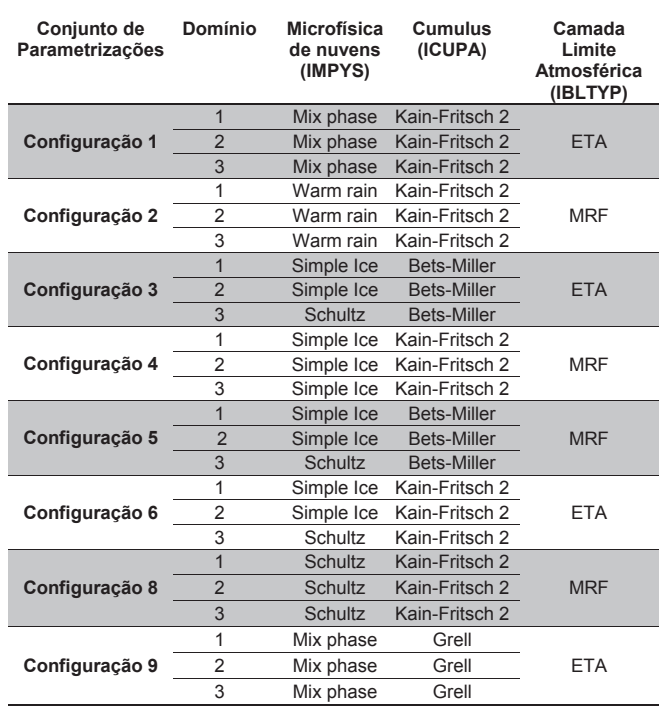

abril de 2010 às $21 \mathrm{~h}$ (00Z do dia 05) e integrado até o dia 06 de abril de 2010 às $21 \mathrm{~h}$ (00Z do dia 07) utilizando como condições de fronteira lateral as previsões do modelo GFS (Sistema de Previsão Global) com resolução espacial de $0.5^{\circ}$ $(\sim 55 \mathrm{~km})$; na rodada 2 o modelo foi inicializado a partir do dia 05 de abril de 2010 às $21 \mathrm{~h}(00 \mathrm{Z}$ do dia 06) e integrado até o dia 07 de abril de 2010 às $21 \mathrm{~h}(00 \mathrm{Z}$ do dia 8$)$, utilizando como condições de fronteira lateral as previsões do modelo GFS com resolução espacial de $1^{\circ}(\sim 110 \mathrm{~km})$. Nas duas rodadas os dados tinham resolução temporal de 3 horas.

A análise foi realizada utilizando o campo de precipitação e as previsões foram geradas a cada 3 horas (horizonte da previsão). Os resultados foram comparados com dados observados de precipitação das estações pluviométricas da GeoRio. Primeiramente foram comparadas a precipitação simulada acumulada em $24 \mathrm{~h}$ com os dados observados, realizando uma avaliação qualitativa dos resultados de cada conjunto de parametrizações, principalmente em relação aos aspectos de intensidade e localização espacial da precipitação, para em seguida, escolher o conjunto que melhor representou o evento, a fim de comparar a precipitação acumulada observada e prevista a cada 3 horas, durante o evento. Por fim, as previsões desse mesmo conjunto de parametrizações foram comparadas com os dados observados, porém com condições iniciais distintas.

\section{Resultados}




\subsection{Comparação entre os conjuntos de parametrização e a chuva observada}

Os resultados obtidos com o modelo MM5 na primeira rodada, inicializada às $00 \mathrm{Z}$ do dia 05 , referentes à chuva acumulada em $24 \mathrm{~h}(09 \mathrm{~h}$ do dia 05/04 às $09 \mathrm{~h}$ do dia 06/04) de todos os conjuntos de parametrizações empregados no estudo estão na Figura 6, e a precipitação em 24h medida pelos pluviômetros da Geo-Rio está na Figura 7. O retângulo tracejado nos mapas resultantes das simulações representa a cidade do Rio de Janeiro.

Observa-se que, de um modo geral, a maior parte dos esquemas subestimaram a precipitação ocorrida que ultrapassou os $180 \mathrm{~mm}$. Esse evento ocasionou valores de precipitação extremamente altos, acima da média do mês de abril.

Os conjuntos de parametrizações que obtiveram pior desempenho nas simulações foram os conjuntos P4 e P9, identificando pouca quantidade de chuva na cidade do Rio de Janeiro (variando de 5 a $70 \mathrm{~mm}$ ). O conjunto P4 é o único que possui uma parametrização de microfísica somente com "Simple Ice" nas três grades. Já o conjunto P9 é o único que possui a configuração de cumulus utilizando "Grell".

Os conjuntos de parametrizações P1 e P8 obtiveram bom desempenho identificando uma elevada quantidade de precipitação na cidade do Rio de Janeiro, principalmente na região da Barra e Recreio, com totais de chuva em torno de $180 \mathrm{~mm}$. Porém, o conjunto que representou de forma bastante realística o evento foi o P5, simulando mais de $180 \mathrm{~mm}$ de precipitação nas Zonas Sul e Norte do Rio de Janeiro, diminuindo um pouco a quantidade de chuva na direção Oeste. Além disso, esse conjunto foi o único que conseguiu simular uma precipitação alta nos municípios de Niterói e São Gonçalo, fato que ocorreu, ocasionando muitas mortes e acidentes nessa região.

Ao observar a Tabela 2 é possível notar que o conjunto de parametrizações P5 é praticamente igual ao conjunto P3, somente com a modificação da Camada Limite Atmosférica. No P5 se utiliza MRF e no P3, ETA. Dessa forma, pode-se afirmar que a simulação foi sensível também à escolha da parametrização da camada limite. Com essa configuração de parametrizações, conclui-se que, considerando a configuração ETA para a camada limite, o modelo simula uma maior precipitação em regiões com topografia elevada do que nas baixadas, assim como já está reportado por ONS (2013), que utiliza esta mesma parametrização.

Comparando os conjuntos de parametrizações P3 e P6, percebe-se que ambos apresentam as mesmas parametrizações, modificando somente a parametrização de cumulus: o P3 utiliza "Bets-Miller" e o P6, "KainFritsch 2". Pode-se inferir que em ambos os casos, a localização dos máximos e mínimos de precipitação está parecida, porém, nas simulações com o conjunto P3 verifica-se uma maior quantidade de chuva nos pontos de máximo, com núcleos de chuva ultrapassando $180 \mathrm{~mm}$.

Já comparando os conjuntos de parametrizações $\mathrm{P} 1$ e P9, a única diferença entre eles está também na parametrização de cumulus: P1 utiliza "Kain-Fritsch 2" e P9, "Grell". A diferença entre os dois resultados é significativa, enquanto o P1 identifica uma magnitude considerável de precipitação na região, mostrando alguns núcleos de chuva com intensidade de até $160 \mathrm{~mm}$, o conjunto P9 praticamente não simulou precipitação. Ou seja, a parametrização de "Grell" aparentemente não se mostrou adequada para simular eventos de convecção rasa, o que vem de acordo com o relatado por Grell (1993), onde o autor ressaltou a importância de se concentrar esforços para melhor representar a convecção rasa nos esquemas de convecção.

Uma última comparação foi feita com os conjuntos P2, P4 e P8, com a diferença na parametrização de microfísica das nuvens. No conjunto P2 foi utilizado o esquema "Warm Rain", no P4 "Simple Ice" e no P8 "Shultz". Os esquemas de "Warm Rain" e "Simple Ice", nesse caso, não simularam corretamente a magnitude da precipitação, em comparação com o esquema "Schultz", o qual detectou intensa precipitação em todo o município do Rio de Janeiro, com intensidades chegando a $160 \mathrm{~mm}$ na maior parte da região, corroborando o discutido em Schultz (1995), onde foi abordado que este esquema representaria melhor o fenômeno de convecção rasa do que os esquemas que o antecederam. 


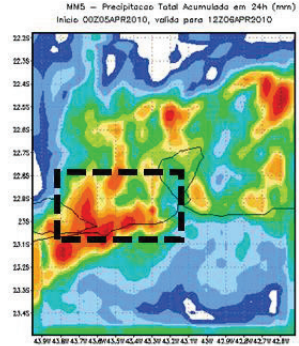

(A) P1

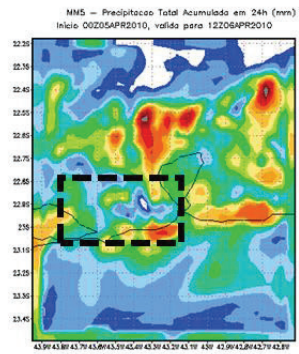

(B) P2

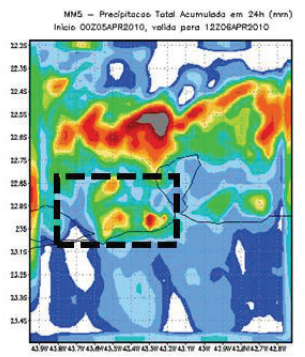

(C) P3

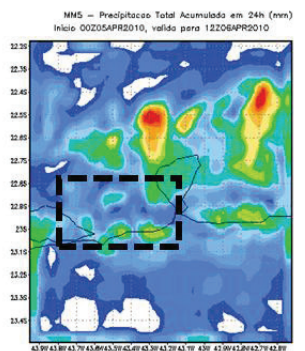

(D) P4

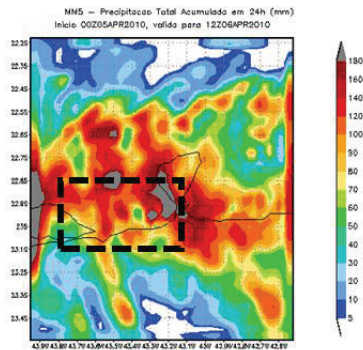

(E) P5

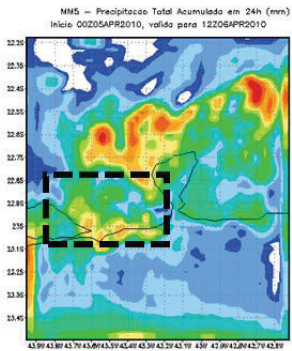

(F) P6

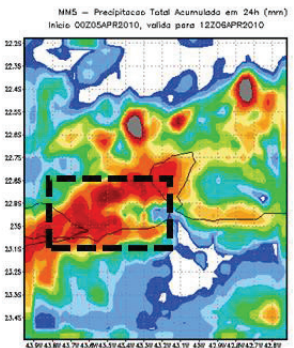

(G) P8

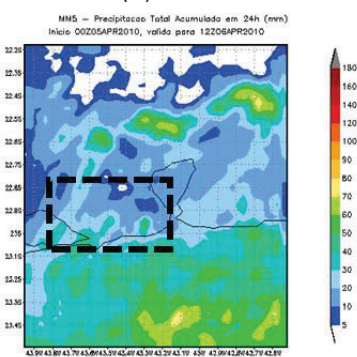

(H) P9

Figura 6 - Resultados das simulações no MM5, rodada 1, da precipitação acumulada em 24h, dos oito grupos de parametrizações

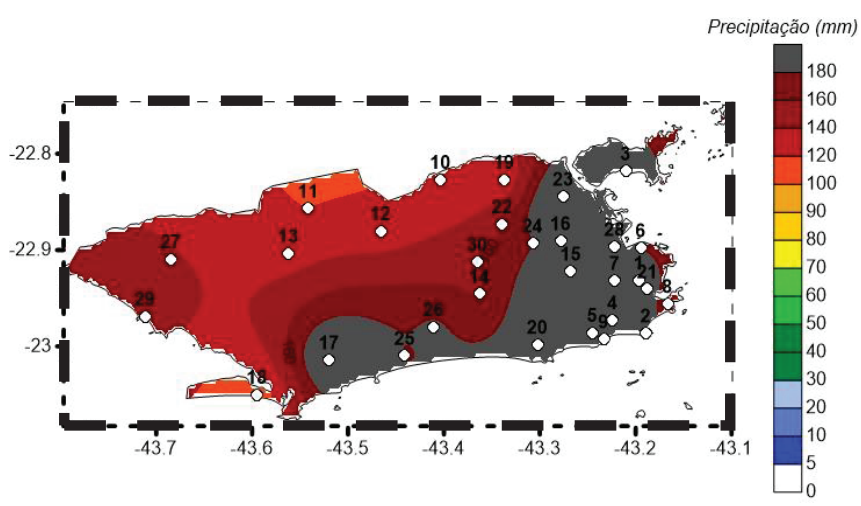

Figura 7- Precipitação acumulada em 24h através dos dados dos pluviógrafos 


\subsection{Resultados da previsão do conjunto P5}

Como o conjunto de parametrizações P5 apresentou melhores resultados na simulação da precipitação acumulada em $24 \mathrm{~h}$, da rodada 1 , este conjunto foi escolhido para a análise de todos os resultados das simulações feitas na grade $3(3 \mathrm{~km})$, de maior resolução. A Figura 8 mostra os resultados das simulações do MM5 (esquerda) e as respectivas precipitações observadas das $00 \mathrm{~h}$ do dia 05/04/2010 até as $03 \mathrm{~h}$ do dia 06/04/2010 (direita),

Analisando as simulações, observa-se que logo nas primeiras três horas de simulação (Figura 8a) as previsões do modelo já indicavam chuva na cidade do Rio de Janeiro, o que não aconteceu na realidade. Isso provavelmente se deve a fase de "spin-up" (tempo de ajuste do modelo).
Após a fase de "spin-up", o modelo volta a simular chuva na cidade do Rio de Janeiro em torno nas 9 h da manhã do dia 05/04 (Figura 8d), adiantando a precipitação real que começa em torno de $12 \mathrm{~h}$ (Figura 8e). A partir desse horário a precipitação permanece na região. É interessante observar também que a grande quantidade de chuva acumulada na região Norte do Rio até às $18 \mathrm{~h}$ do dia 05 (Figura 8g), simulada pelo modelo, aconteceu na verdade das $18 \mathrm{~h}$ às $21 \mathrm{~h}$ (Figura $8 \mathrm{~h}$ ). Assim, observa-se, através desses exemplos, que houve uma tendência do modelo em antecipar a precipitação em cerca de três horas, o que é razoável para um modelo de mesoescala. Porém, ao analisar a Figura 8h, observa-se que a posição e a intensidade do núcleo de precipitação mais intenso foram capturadas pela simulação do mesmo horário.
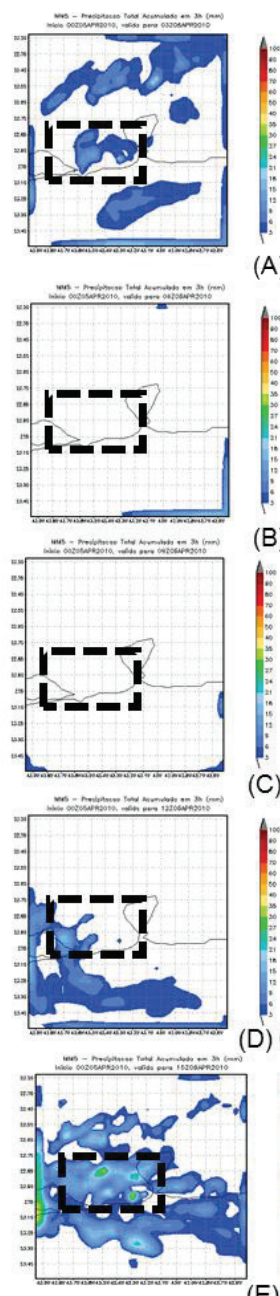

(D) $05 / 04-09 h$

(B) $05 / 04-03 \mathrm{~h}$

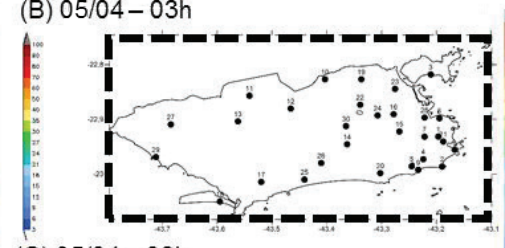

(C) $05 / 04-03 \mathrm{~h}$
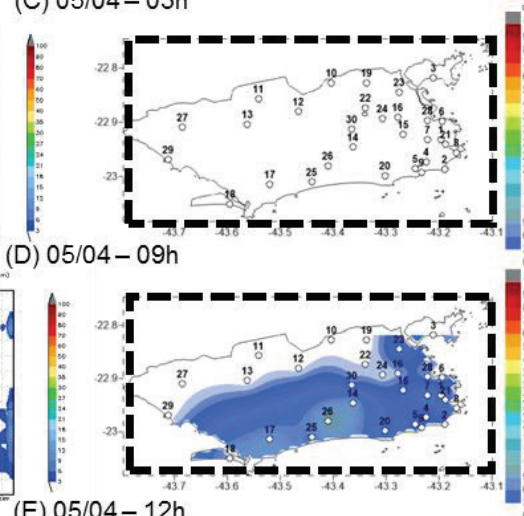
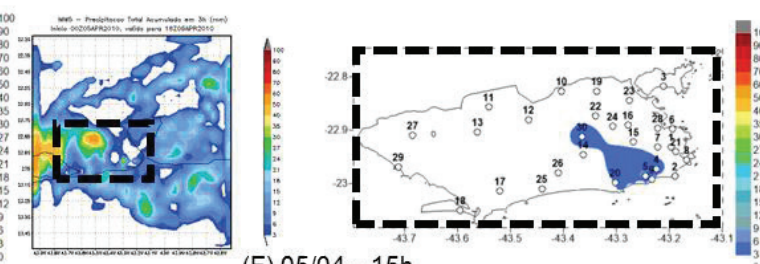

(F) $05 / 04-15 \mathrm{~h}$
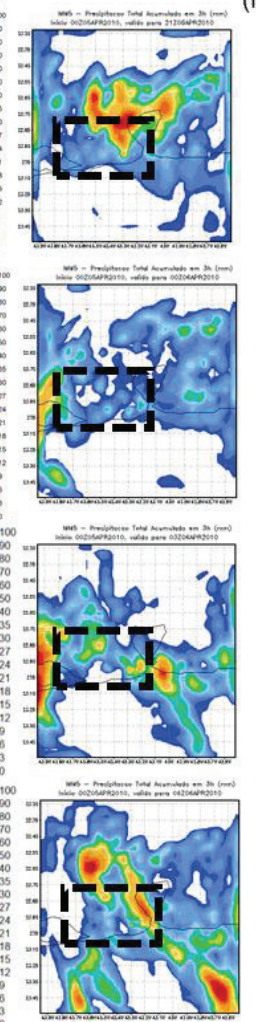

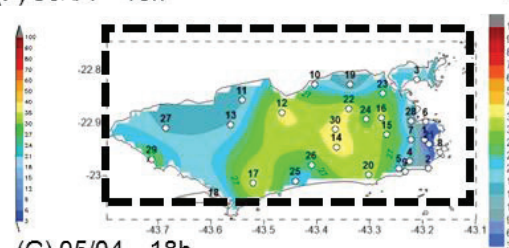

(G) $05 / 04-18 \mathrm{~h}$

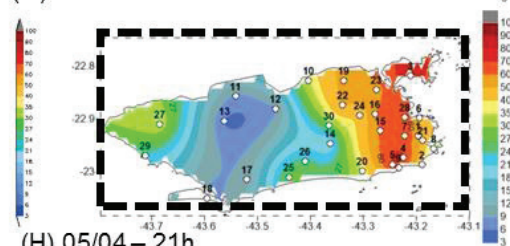

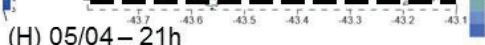

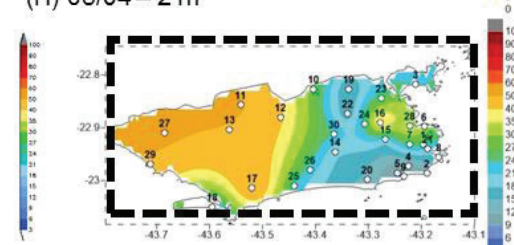

(I) $06 / 04-00 \mathrm{~h}$

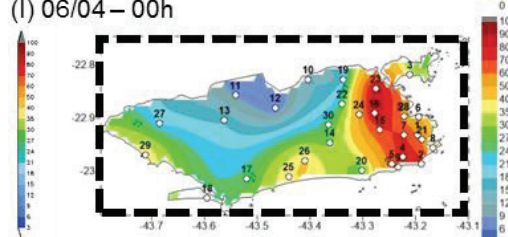

(J) $06 / 04-03 h$

Figura 8 - Previsão do conjunto de parametrizações P5 - Grade 3 e comparação com a chuva observada pelas estações pluviométricas. Horário local 


\subsection{Comparação entre as duas rodadas com condições iniciais distintas}

As duas simulações iniciadas em diferentes horários são comparadas em um período de tempo comum, das $00 \mathrm{~h}$ até às $09 \mathrm{~h}$ do dia 06 de abril, utilizando a Grade 3 do conjunto de parametrizações P5 (Figura 9).

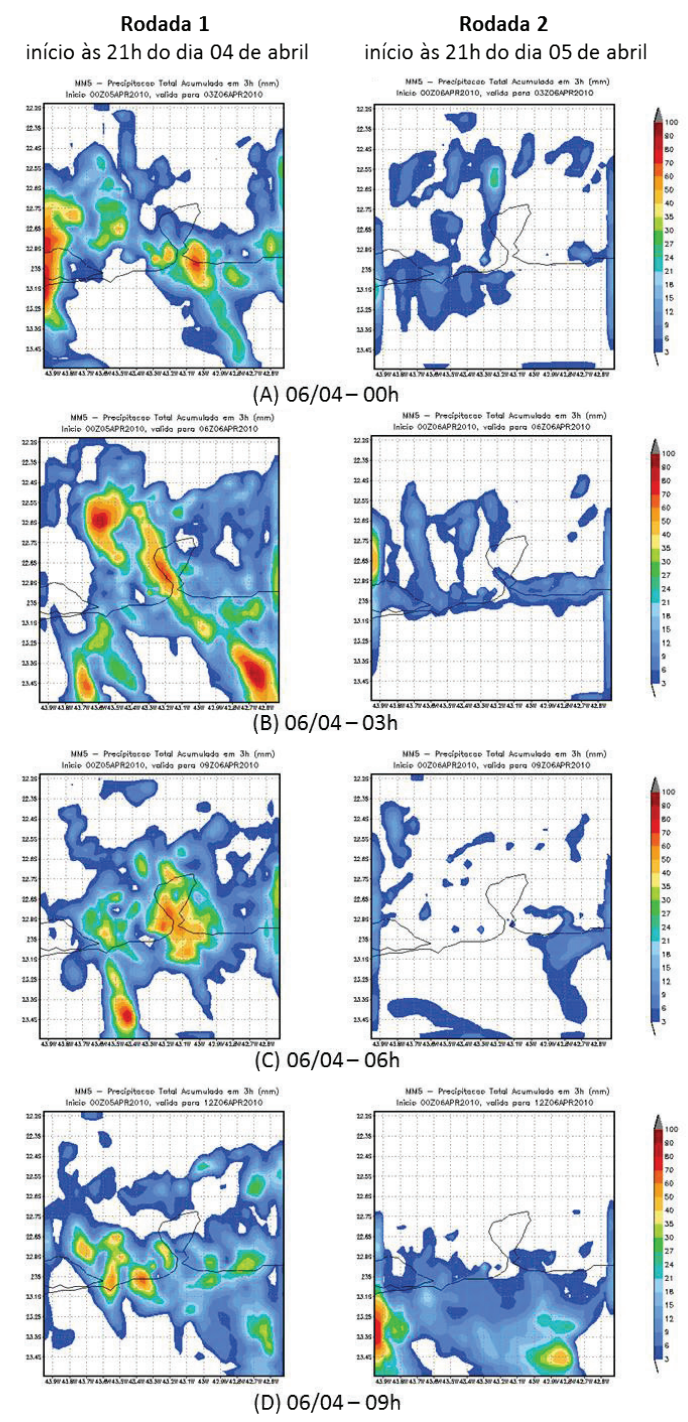

Figura 9 - Comparação entre as duas rodadas realizadas utilizando o conjunto P5 de

parametrizações na grade 3 , de $00 \mathrm{~h}$ às $09 \mathrm{~h}$ do dia 6 de abril. Horário local

No MM5 as condições iniciais só incluem a temperatura, os campos de vento, a pressão reduzida ao nível do mar e a umidade especifica.

Todas as outras variáveis, como por exemplo, a água líquida das nuvens, são inicializadas como zero (COSTA e SOUSA, 2002). Assim, como a rodada 2 está iniciando justamente no horário no qual se observa uma grande quantidade de precipitação, a simulação vai demorar até conseguir captar água líquida nas nuvens (período de "spin-up"), diminuindo assim a magnitude da chuva ocorrida, como é observado nos resultados da rodada 2 .

Para uma possível diminuição desse tempo de ajuste do modelo seria necessário fazer um estudo utilizando a assimilação de dados de precipitação observados durante as simulações, conduzindo o modelo para condições mais próximas da realidade.

Além disso, existe a diferença entre as resoluções espaciais dos dados adquiridos do modelo GFS, utilizados como condição de contorno, a saber: para a simulação iniciada no dia 04/04 (Rodada 1) foram utilizadas condições de contorto do GFS com resolução espacial de $0.5^{\circ}$, e para a simulação inicializada no dia 05/04 a resolução espacial das condições de contorno foi de $1^{\circ}$. Porém, não é possível dizer se esta diferença alterou a qualidade dos resultados.

\section{Conclusões}

O evento de chuva ocorrido nos dias 05 e 06 de abril de 2010 foi um evento de longa duração que causou muitas perdas humanas e econômicas, principalmente nas cidades do Rio de Janeiro e Niterói, chovendo entre a tarde do dia 05 e a manhã do dia 06 de abril, em média, $178 \mathrm{~mm}$, o dobro da média de abril no Rio de Janeiro.

Através das simulações pode-se concluir que a maior parte dos conjuntos subestimou a precipitação ocorrida, porém o conjunto P5 foi o que obteve melhores resultados, representando bem a magnitude e a distribuição espacial do evento. Este conjunto possui esquema de microfísica Simple Ice (Grades 1 e 2) e Schultz (Grade 3), esquema de cumulus Bets-Miller e esquema MRF para camada limite atmosférica. Ao compará-lo com o conjunto P3, é possível observar que só existe uma diferença de parametrização, sendo esta a da camada limite, modificando sensivelmente os resultados.

Pode-se dizer que os diferentes resultados dos outros conjuntos de parametrização mostram a incerteza inerente à escolha do conjunto de parametrização mais adequado 
Além disso, não se pode afirmar que somente pelo fato do conjunto P5 ter se mostrado a melhor opção na simulação deste evento meteorológico no município do Rio de Janeiro, ele vai ser eficiente para prever outros fenômenos meteorológicos que também podem afetar a cidade. Configurações de esquemas paramétricos que apresentam bons resultados em um evento específico, como eventos de convecção rasa, nesse caso, podem não ter o mesmo desempenho para simular convecção profunda.

Existem grandes diferenças entre os resultados de cada conjunto de parametrização, sendo difícil e ineficiente escolher somente um conjunto para se utilizar na hora de tomar decisões. Uma opção é o cálculo de uma media desses conjuntos de previsões (ensemble), visando minimizar o erro das simulações e podendo inclusive atribuir diferentes pesos para cada conjunto de parametrizações, dependendo do tipo de sistema atmosférico a ser simulado.

Por último, através da comparação das simulações geradas pelas duas rodadas do conjunto de parametrizações P5, foi possível notar uma menor qualidade das simulações da rodada 2, devido a inicialização da rodada durante a ocorrência da chuva e ao tempo de ajuste do modelo (spin-up), o que expõe de forma efusiva a necessidade de se investir na assimilação de dados para previsões de curtíssimo prazo.

Pelo que foi discutido neste estudo, observase também a dificuldade em se determinar de forma definitiva a resolução, a inicialização e as parametrizações do modelo MM5 que melhor atendem às necessidades de simulação da precipitação em uma determinada região, sendo necessário, portanto, a realização de vários estudos como este, de modo a se definir as melhores configurações do modelo para a representação de eventos de precipitação intensa na cidade do Rio de Janeiro, por exemplo.

Porém, de uma maneira geral, o modelo mostrou potencial para simular este evento de precipitação, mostrando-se como uma ferramenta útil para prever e diminuir as consequências dos eventos de chuvas intensas no município do Rio de Janeiro.

\section{Referências}

AMENGUAL, A., ROMERO, R., \& ALONSO, S. (2008). Hydrometeorological ensemble simulations of flood events over a small basin of Majorca Island, Spain. Quarterly Journal of the Royal Meteorological Society, 134(634), 12211242.

BRAY, M., HAN, D., XUAN, Y., BATES, P., \& Williams, M. (2011). Rainfall uncertainty for extreme events in NWP downscaling model. Hydrological Processes, 25(9), 1397-1406.

COELHO, F. G., DA HORA, M. A. G. M., DA HORA, A. F. (2010). The storms in Rio de Janeiro City in April 2010. Engevista, 13 (1), 57-62.

MACHADO, C. O. (2009). Climatologia da precipitação no município do Rio de Janeiro. Revista Brasileira de Meteorologia, 24(1), 24-38.

COSTA E SOUSA, T. A. S. (2002). Previsão Meteorológica em Portugal Continental utilizando o modelo operacional e de investigação MM5. M. Sc. Universidade Técnica de Lisboa, Instituto Superior Técnico. Lisboa, Portugal. 120p.

DODLA, V. B. R.; RATNA, S. B. (2010). Mesoscale characteristics and prediction of an unusual extreme heavy precipitation event over India using high resolution mesoscale model. Atmospheric Research, v. 95, 255-269.

DUDHIA, J., GILL, D., MANNING, K., BOURGEOIS, A., WANG and BRUYERE, C. (2002). PSU/NCAR Mesoscale Modeling System Tutorial Class Notes and Users' Guide (MM5 Modeling SystemVersion 3).

FERNÁNDEZ, J., MONTÁVEZ, J. P., SÁENZ, J., GONZÁLEZ-ROUCO, J. F., \& ZORITA, E. (2007). Sensitivity of the MM5 mesoscale model to physical parameterizations for regional climate studies: Annual cycle. Journal of Geophysical Research: Atmospheres (1984-2012), 112(D4).

GRELL, G. (1993). Prognostic evaluation of assumptions used by cumulus parameterizations. Monthly Weather Review, 121, 764-787. 
GRELL, G. A., DUDHIA J., STAUFFER D. R. (1994). A description of the fifth generation Penn State/NCAR mesoscale model (MM5), NCAR Technical Note. National Center for Atmospheric Research, Boulder.

JEREZ, S., MONTAVEZ, J. P., GOMEZNAVARRO, J. J., LORENTE-PLAZAS, R., GARCIA-VALERO, J. A., \& JIMENEZGUERRERO, P. (2013). A multi-physics ensemble of regional climate change projections over the Iberian Peninsula.Climate dynamics, 41(7-8), 1749-1768.

ONS (Operador Nacional do Sistema Elétrico). Aprimoramento da Metodologia de remoção de viés da previsão de precipitação - aplicação na bacia do Alto Rio São Francisco. Nota técnica, 2013.

OYAMA, M. D. (2006). Erros sistemáticos no clima da região tropical da América do Sul simulado pelo modelo regional MM5 em baixa resolução horizontal. Revista Brasileira de Meteorologia, 21(1), 118-133.

PADILHA, S. F. (2011). Simulações de Eventos de Chuvas Intensas no Estado do Rio de Janeiro usando o Modelo WRF. Dissertação de Mestrado. Universidade Federal do Rio de Janeiro, Rio de Janeiro.
QUEEN, A., ZHANG, Y. (2008). Examining the sensitivity of MM5- CMAQ predictions to explicit microphysics schemes and horizontal grid resolutions, Part I-database, evaluation protocol, and precipitation predictions, Atmos. Environ., 42, 3842-3855.

REBELlO, E. R. G., CARVAlHO, B. E. F. C., COSTA, J. A. V., FREITAS, M. A. S., CAVALCANTE, O. A. (2012) Diagnóstico, Monitoramento e Prognóstico das Chuvas Intensas no Rio de Janeiro no período de 06 a 09 de Abril de 2010. In: Congresso Brasileiro Sobre Desastres Naturais, 2012, Rio Claro - SP. Anais do Congresso Brasileiro Sobre Desastres Naturais. Rio Claro: Editora da Unesp.

SCHULTZ, P. (1995): An explicit cloud physics parameterization for operational numerical weather prediction, Mon. Weather Rev., 123, 3331-3343.ZHANG, Y., CHENG, S. H., CHEN, Y. S., \& WANG, W. X. (2011). Application of MM5 in China: Model evaluation, seasonal variations, and sensitivity to horizontal grid resolutions. Atmospheric Environment, 45(20), 3454-3465. 